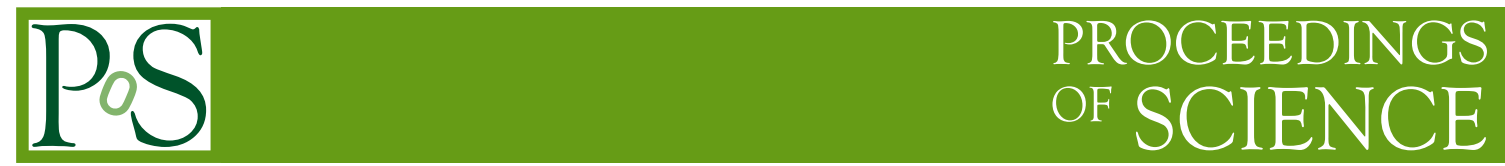

\title{
Running neutrino Mass from Six Dimensions
}

\author{
Sudhir Vempati*† \\ Centre for High Energy Physics, Indian Institute of Science \\ Bangalore 560 012, India \\ E-mail: vempati@ets.iisc.ernet.in
}

In the present talk, we will discuss a six dimensional mass generation for the neutrinos. The SM neutrinos live on a 3-brane and interact via a brane localised mass term with a Weyl singlet neutrino residing in all the six dimensions. We present the physical neutrino mass spectrum and show that the active neutrino mass and the KK masses have a logarithmic cut-off dependence at the tree level. This translates in to a renormalisation group running of $v$-masses above the KK compactification scale coming from classical effects without any SM particles in the spectrum. This could have effects in neutrinoless double beta decay experiments.

From Strings to LHC

January 2-10 2007

International Centre Dona Paula, Goa India

\footnotetext{
* Speaker.

${ }^{\dagger}$ This work is done in collaboration with E. Dudas and C. Grojean.
} 


\section{In a nut shell}

Extra dimensional models have become quite popular in the past few years with the realisation that they could exist at far lower scales compared to the Planck scale, $M_{p l}$, and thus they could have an impact at the TeV scale physics [1,2,3]. Some of the significant implications could be for the gauge coupling unification [4], solutions to the hierarchy problem [1,3] each having its own phenomenological impact like for example, collider searches [6] and precision measurements of standard electroweak parameters [5].

The existence of the extra spacetime dimensions at some scale closer or higher than the weak scale also gives much scope to build models related to fermion masses and mixings, particularly for light neutrinos [7], qualitatively rather different from the traditional 4D seesaw mechanism [8]. In this case, a singlet neutrino (right handed) could be allowed to propagate in the bulk whereas the SM (left-handed) neutrino would be confined to the 3-brane, leading to new possibilities of neutrino masses and mixing $[9,10,11]$. While this possibility has been explored in detail both in model building as well as in terms of phenomenological analysis [12], most of these studies have been confined to the case where there is only one additional dimension. It is necessary to extend these analysis for higher number of extra dimensions. This is because new features could arise in higher dimensional field theories which could give rise to different phenomenology. In particular, interesting 6D models have been constructed to break the electroweak symmetry through nontrivial Wilson lines [13], to guarantee the proton stability up to dimension fifteen operators [14], to predict the number of chiral generations [15], to provide a dark matter candidate [16], to construct realistic GUT models [17] etc. 6D models have also been constructed to reproduce the neutrino mass spectrum [18].

In the present talk, I will discuss about our work[19] where we have studied the case of neutrino masses in a six dimensional model. In six dimensional models, it has been known for some time that orbifold compactification produces some peculiar properties such as 'tree level' renormalisation of coupling constants [20,21]. While this has been known for the scalar case for some time, we have studied this property in detail for the case of neutrinos with a brane localized mass term on a $T^{2} / \mathbb{Z}_{2}$ orbifold. We found that the physical neutrino mass (and also higher Kaluza-Klein modes) have a logarithmic divergence related to the brane thickness.

The interpretation of this result is similar to the brane-localized scalar mass term discussed in [21] and it can be renormalised in a similar manner by adding a neutrino Dirac mass brane counterterm. As a result, the neutrino mass runs, more precisely increases with energy, above the compactification scale $R^{-1}$, where for simplicity we consider the case of two equal radii. For radii in the $\mathrm{eV}$ to $\mathrm{MeV}$ range, the effect of this classical running has no counterpart in four dimensions, since it arises without the presence of any new particle charged under the Standard Model gauge group. This can be tested in processes with off-shell neutrinos, like the neutrinoless double beta decay, where the increase in the neutrino mass at $\mathrm{GeV}$ energies enhances the amplitude of the process.

\section{The Setup}

We have considered a six dimensional space, the two extra dimensions being compact. The 
compact space is orbifolded on $T^{2} / Z_{2}$, which acts as an reflection $\left(y_{1}, y_{2}\right) \rightarrow\left(-y_{1},-y_{2}\right)$, with $y_{i}$ representing the two extra dimensions. This orbifold has four fixed points. These and corresponding $\mathbb{Z}_{2}$ transformations of the coordinates around the fixed points are summarised as:

$$
\begin{array}{cccc}
y_{1} \rightarrow-y_{1} & y_{1} \rightarrow-y_{1}+2 \pi R_{1} & y_{1} \rightarrow-y_{1} & y_{1} \rightarrow-y_{1}+2 \pi R_{1} \\
y_{2} \rightarrow-y_{2} & y_{2} \rightarrow-y_{2} & y_{2} \rightarrow-y_{2}+2 \pi R_{2} & y_{2} \rightarrow-y_{2}+2 \pi R_{2} \\
(0,0) & \left(\pi R_{1}, 0\right) & \left(0, \pi R_{2}\right) & \left(\pi R_{1}, \pi R_{2}\right) .
\end{array}
$$

Finally, lets also note that in complex notation, the action of $\mathbb{Z}_{2}$ on the compact space is a twodimensional $\pi$ rotation, $Z_{2}\left(y_{1}+i y_{2}\right)=e^{i \pi}\left(y_{1}+i y_{2}\right)$.

\subsection{The scalar case}

Before proceeding to the neutrino case, its instructive to study the scalar case as it is much easier to understand the physics. Further, as a starting point, let us set the bulk mass to zero and add a localized mass term at the origin of the $2 \mathrm{D}$ compact space. The corresponding action reads ${ }^{1}$ :

$$
S=\frac{1}{2} \int d^{4} x d^{2} y\left(\left(\partial^{M} \Phi\right)\left(\partial_{M} \Phi\right)-h_{2} \Phi^{2} \delta^{2}(\mathbf{y})\right)
$$

where $h_{2}>0$ is a dimensionless coupling in the natural 6D units for which the scalar field $\Phi$ has dimension two. The coupling $h_{2}$ is localized at the origin of the compact space. The field equation is free in the bulk and has a delta function source at the origin

$$
\partial_{M} \partial^{M} \Phi+h_{2} \Phi \delta^{2}(\mathbf{y})=0
$$

The KK spectrum and eigenvalues:

If the scalar field $\Phi$ is even under the orbifold action, it can be decomposed on a complete basis formed by the cosine functions:

$$
\Phi(x, \mathbf{y})=\sum_{\left(k_{1}, k_{2}\right) \in \mathscr{I}}\left\langle y_{1}, y_{2} \mid k_{1}, k_{2}\right\rangle \phi_{\left(k_{1}, k_{2}\right)}(x)
$$

with

$$
\left\langle y_{1}, y_{2} \mid k_{1}, k_{2}\right\rangle=\frac{1}{\sqrt{2 \pi^{2} R_{1} R_{2}}} \frac{\cos \left(\frac{k_{1}}{R_{1}} y_{1}+\frac{k_{2}}{R_{2}} y_{2}\right)}{\sqrt{2^{\delta_{k_{1} 0} \delta_{k_{2} 0}}}},
$$

The indices $k_{1,2}$ belong to the set $\mathscr{I}$

$$
\mathscr{I}=\{(0 ; 0),(1 \ldots \infty ; 0),(0 ; 1 \ldots \infty),(1 \ldots \infty ; 1 \ldots \infty),(1 \ldots \infty ;-\infty \ldots-1)\}
$$

The scalar action (2.2) then takes the following form after integration over the two extra dimensions

$$
\mathscr{L}=\mathscr{L}_{k i n}-\frac{1}{2} \sum_{\left(k_{1}, k_{2}\right) \in \mathscr{I}}\left(\frac{k_{1}^{2}}{R_{1}^{2}}+\frac{k_{2}^{2}}{R_{2}^{2}}\right) \phi_{\left(k_{1}, k_{2}\right)}^{2}-\frac{\bar{m}^{2}}{2}\left(\sum_{\left(k_{1}, k_{2}\right) \in \mathscr{I}} \frac{\sqrt{2}}{\sqrt{2^{\delta_{k_{1} 0} \delta_{k_{2} 0}}}} \phi_{\left(k_{1}, k_{2}\right)}\right)^{2},
$$

\footnotetext{
${ }^{1}$ We are using a $(+,-,-,-,-,-)$ metric. The index $M$ denotes bulk coordinates and runs from $0,1,2,3,5,6$, while $\mu=0,1,2,3$ denotes brane coordinates.Finally, $\partial_{1,2}$ is a short-handed notation for $\partial_{y_{1,2}}$
} 
where

$$
\bar{m}^{2} \equiv \frac{h_{2}}{4 \pi^{2} R_{1} R_{2}}
$$

is the naive (volume suppressed) four dimensional lightest scalar mass, which is typically of the order and slightly smaller than the compactification mass scale. The mass term of the 4D action (2.7) is

$$
\mathscr{L}_{\text {mass }}=-\frac{1}{2} \sum_{\left(k_{1}, k_{2}\right),\left(p_{1}, p_{2}\right) \in \mathscr{I}} \phi_{\left(k_{1}, k_{2}\right)} \mathscr{M}_{\left(k_{1}, k_{2}\right),\left(p_{1}, p_{2}\right)}^{2} \phi_{\left(p_{1}, p_{2}\right)}
$$

with the mass matrix given by

$$
\mathscr{M}_{\left(k_{1}, k_{2}\right),\left(p_{1}, p_{2}\right)}^{2}=\frac{2 \bar{m}^{2}}{\sqrt{2^{\delta_{k_{1} 0}} 2^{\delta_{k_{2} 0}}}}+\left(\frac{k_{1}^{2}}{R_{1}^{2}}+\frac{k_{2}^{2}}{R_{2}^{2}}\right) \delta_{k_{1}, p_{1}} \delta_{k_{2}, p_{2}} .
$$

The diagonalization of this mass matrix will define the KK mass eigenstates. The eigenvalues and eigenvectors of the mass matrix (2.10) are given by the characteristic equation

$$
\mathscr{M}^{2} \Psi_{m}=m^{2} \Psi_{m}
$$

where $m^{2}$ represents the eigenvalues and $\Psi$ is the eigenvector in the basis $\left|k_{1}, k_{2}\right\rangle_{\left(k_{1}, k_{2}\right) \in \mathscr{I}}$ defined in the previous section $\left(\Psi_{\left(k_{1}, k_{2}\right)}=\left\langle k_{1}, k_{2} \mid \Psi_{m}\right\rangle\right)$. This leads to the eigenvalue equation after some algebra:

$$
\frac{1}{\bar{m}^{2}}=\sum_{k_{1}, k_{2}=-\infty}^{\infty} \frac{1}{m^{2}-k_{1}^{2} / R_{1}^{2}-k_{2}^{2} / R_{2}^{2}}
$$

Note that, here, the sums have been conveniently rewritten from $k_{i}=-\infty$ to $+\infty$. From this equation, we can obtain an estimate for the lightest eigenvalue in the large radii limit $\left(m<R^{-1}\right)$. This is given by :

$$
\frac{1}{m^{2}}=4 \pi^{2} R_{c}^{2}\left(\frac{1}{h_{2}}+\frac{1}{4 \pi} \ln \left(\Lambda^{2} R_{c}^{2}\right)\right)
$$

where we have introduced the logarithmic cut-off $\Lambda$ and also considered equal radii limit for simplicity $R_{1}=R_{2}=R_{c}, R_{c}$ denoting the compactification radius. The interpretation of the logarithmic divergence in the sum, which in turn enters our eigenvalue equation can be understood by realising that the brane localised couplings do run in the sense of four dimensional renormalisation group running[20,21]. The coupling $h_{2}$ is interpreted as the value of the coupling at the high cut-off scale, $\Lambda$. For energies lower than the cut-off scale and larger the compatification scale, the running of the mass eigenvalue can be observed. Below the compactification scale, there is no running present and the eigenvalue just takes a constant profile in energy.

\section{The Case of the Neutrino in $6 \mathrm{D}$}

Let us now concentrate on the case of a neutrino in a 6D bulk with a brane localised mass term. As we will see soon, the eigenvalue equation in this case will reduce to the one we have already encountered in the scalar case. Before deriving the eigenvalues, let us first describe our framework in more detail. We will assume that the Standard Model fermions are restricted to the 4D brane whereas a singlet $6 \mathrm{D}$ Weyl neutrino is free to propagate in the full six dimensional space. In $6 \mathrm{D}$, 
the Weyl fermion is an eight component spinor. Decomposing it into two four-component 4D Weyl spinors, the orbifold $\mathbb{Z}_{2}$ action becomes

$$
\Psi=\left(\begin{array}{l}
\lambda_{1} \\
\lambda_{2}
\end{array}\right), \quad \mathbb{Z}_{2} \lambda_{1}(\mathbf{y})=\lambda_{1}(-\mathbf{y}), \mathbb{Z}_{2} \lambda_{2}(\mathbf{y})=-\lambda_{2}(-\mathbf{y}) .
$$

This allows us to write a brane localized coupling between the SM left-handed neutrino and the even fermion $\lambda_{1}$ :

$$
\int d^{4} x\left(h \bar{v}_{L} \lambda_{1}(\mathbf{y}=0) H+h . c .\right)
$$

where $H$ is the $4 \mathrm{D}$ Higgs field and $h$ is the Yukawa coupling. The mass dimensions of $\Psi, v_{L}, H$ are respectively $5 / 2,3 / 2$ and 1 . Written in two-component spinor notations, the $6 \mathrm{D}$ lagrangian takes the form :

$$
\begin{aligned}
\mathscr{L}= & -i \lambda_{1} \sigma^{\mu} \partial_{\mu} \bar{\lambda}_{1}-i \lambda_{2} \sigma^{\mu} \partial_{\mu} \bar{\lambda}_{2}+\lambda_{1}\left(\partial_{5}+i \partial_{6}\right) \lambda_{2} \\
& -\bar{\lambda}_{2}\left(\partial_{5}-i \partial_{6}\right) \bar{\lambda}_{1}+g_{2}\left(v_{L} \lambda_{1}+\bar{v}_{L} \bar{\lambda}_{1}\right) \delta^{2}(\mathbf{y}),
\end{aligned}
$$

where, we have now introduced $g_{2}=h\langle H\rangle$ the brane-localized 'Dirac' neutrino mass parameter which actually, in analogy with the scalar case, is a dimensionless parameter. One can easily combine the first order equations of motion from the above lagrangian eq.(3.3) to obtain an uncoupled second order differential equation that is nothing but the Klein-Gordon equation in 6D:

$$
\left(\partial_{\mu} \partial^{\mu}-\partial_{5}^{2}-\partial_{6}^{2}\right) \lambda_{1}(x, \mathbf{y})+g_{2}^{2} \lambda_{1}(x, \mathbf{y}) \delta^{2}(\mathbf{y})=0
$$

and therefore our fermionic problem with brane localized Dirac mass term is reduced to the one of the bulk scalar field with brane localized mass term studied in the previous section. Infact, proceeding in the similar manner as in the scalar case, i.e, by using the KK reduction of the fermionic fields, we can explicitly derive the resultant eigenvalue equation, which has the same form as the one for the scalar case:

$$
\frac{1}{\bar{m}^{2}}=\sum_{k_{1}, k_{2}=-\infty}^{\infty} \frac{1}{m^{2}-k_{1}^{2} / R_{1}^{2}-k_{2}^{2} / R_{2}^{2}} .
$$

\subsection{Majorana Case}

In the previous subsection, we have concentrated on the case where the neutrinos are of Diractype. Let us now consider the Majorana case which requires violation of lepton number. Note that there are two ways to break the lepton number: (a) Breaking the lepton number on the brane by introducing on the brane a lepton number violating mass term for the singlet neutrino; (b) Breaking the lepton number in the bulk through a bulk Majorana mass for the singlet neutrino. We will directly present the eigen value equations of these two cases in the following. Let us first study the case $(a)$ and let us add the lepton number violating mass term $M_{0}$ on the brane

$$
\int d^{4} x d^{2} y\left(M_{0} \lambda_{1} \lambda_{1}+h . c\right) \delta^{2}(\mathbf{y})
$$

Notice that actually from a $6 \mathrm{D}$ perspective $M_{0}$ has mass dimension -1 , whereas after the KK expansion the physical mass parameter is $M_{0} /\left(4 \pi^{2} R_{1} R_{2}\right)$. Similar to the Dirac mass considered 
previously, all phases in the Kaluza-Klein complex masses can be redefined away and have no physical meaning. By a straightforward generalisation of the previous diagonalisation, we find the eigenvalue equation

$$
\frac{1}{\bar{m}^{2}+m M_{0} /\left(4 \pi^{2} R_{1} R_{2}\right)}=\sum_{k_{1}, k_{2}=-\infty}^{\infty} \frac{1}{m^{2}-k_{1}^{2} / R_{1}^{2}-k_{2}^{2} / R_{2}^{2}} .
$$

By considering again for simplicity the case of two equal radii $R_{1}=R_{2}=R_{c}$ and evaluating as before the double sum by keeping the leading IR and UV contributions, we find in the large radii limit

$$
\frac{1}{m^{2}}=4 \pi^{2} R_{c}^{2}\left(\frac{1}{g_{2}^{2}+m M_{0}}+\frac{1}{4 \pi} \ln \left(\Lambda^{2} R_{c}^{2}\right)\right) .
$$

The natural interpretation is again in terms of the running of the physical mass

$$
\frac{1}{m^{2}(\mu)}=\frac{4 \pi^{2} R_{c}^{2}}{\left(g_{2}^{2}+m M_{0}\right)(\mu)}
$$

The case of the brane Majorana mass is the simplest but also the most problematic, since due to the double volume suppression the lepton number violation, is small. The case of the bulk Majorana mass $M$ is more subtle. First of all, a standard Majorana, Lorentz invariant mass in $6 \mathrm{D}, \bar{\Psi}^{C} \Psi$ cannot be written for a Weyl fermion, since it mixes 6D Weyl fermions of opposite chiralities. On the other hand, a Majorana mass term involving only a 6D Weyl fermion, of the form $\bar{\Psi}^{C} \Gamma_{6} \Psi$ can be written, at the expense of breaking the 6D Lorentz symmetry which could be considered as being spontaneously generated by the vev of some vector field. In this case, it is not possible anymore to eliminate the phases in the KK masses by field redefinitions, even for real Majorana mass. Interestingly enough, the interplay between Kaluza-Klein masses and bulk Lorentz violating Majorana mass generates $\mathrm{CP}$ violation. This observation could be related to previous proposals to relate the CP symmetry to discrete subgroups of a higher-dimensional Lorentz group. The eigenvalue equation in this case is

$$
\frac{g_{2}^{2}}{4 \pi^{2} R_{1} R_{2}} \sum_{k_{1}, k_{2}=-\infty}^{\infty} \frac{1}{m-M-i k_{1} / R_{1}+k_{2} / R_{2}}=m .
$$

Let us now try to see the significance of the logarithmic running with some realistic numbers. Fig. 1 plots the running of the neutrino mass-squared with the energy for the Dirac case. We have taken the radii to be of the order of $1 \mathrm{eV}^{-1}$ with the fundamental Planck scale $M_{\star}$ taken to be around $100 \mathrm{TeV}$, in order to recover the 4D Planck scale $M_{P}$ through the standard relation $R_{1} R_{2} M_{\star}^{4}=M_{P}^{2}$ [1]. Note that these numbers are close to the present limits from cosmology on the size of two large extra dimensions. The coupling $g_{2}$ is set to one at the cutoff (microscopic) scale. From the figure, we see that the neutrino mass changes by at least a factor of 3 while running with the radius. Below $R^{-1}$, there is no logarithmic running anymore and the masses keep their values at the compactification scale. This explains the horizontal line below $R^{-1}$. We interpret this result as follows. The pole of the propagator, defines the physical mass of the neutrino which is equivalent to the 'running' eigenvalue evaluated at $R^{-1}$. As long as neutrinos are 'on-shell', they carry this mass. The 'running' neutrino masses on the other hand varies with the off-shell momentum. Experimentally, the effect of this 'running' could be possibly seen in processes where 


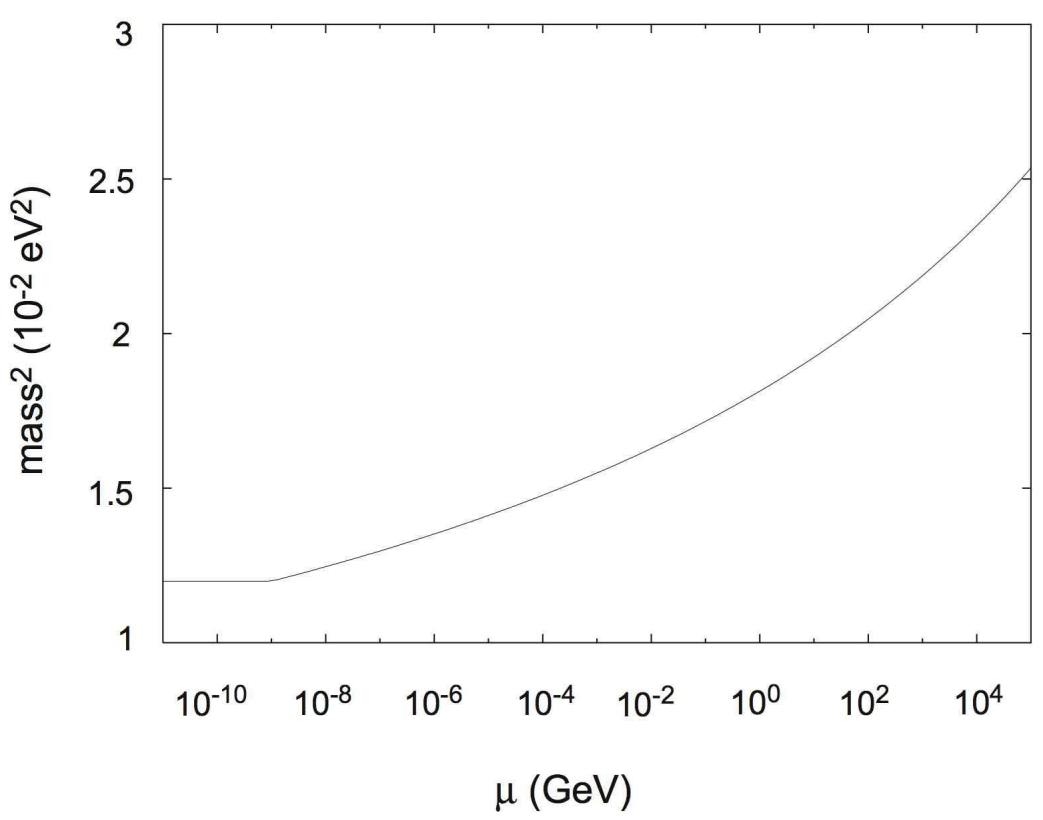

Figure 1: Running of the neutrino squared masses with the energy. We have chosen $\Lambda=10^{5} \mathrm{GeV}, g_{2}=1$, and $R_{1}=R_{2}=1 \mathrm{eV}^{-1}$. The running occurs between the cutoff scale and the compactification scale. Below the compactification scale, there is not running anymore.

neutrinos are off-shell. The best probed process in this regard is neutrinoless double beta decay which involves lepton number violating neutrino masses. We note only that as long as the lepton number is violated by Majorana masses and the running of the Dirac masses does occur, this is enough to have observable effects due to the running between the pole mass and the off-shell momentum, of the order the nucleon mass, in the neutrinoless double beta decay diagrams.

\section{Neutrino oscillations}

Lets now consider the interesting phenomena of neutrino oscillations. Generally, the gauge neutrino eigenstates $v_{f}$ are related to the set of mass eigenstates $\tilde{v}_{i}$ through a unitary mixing matrix $U$ as

$$
v_{f}=\sum_{i} U_{f i} \tilde{v}_{i},
$$

where the matrix $U$ is extracted from the neutrino mass matrix. The probability of oscillation between two gauge eigenstates $v_{f}$ and $v_{f}^{\prime}$ after a time $t$ is given by

$$
P_{f \rightarrow f^{\prime}}(t)=\sum_{i}\left|U_{f i} U_{f^{\prime} i}\right|^{2}+2 \sum_{i>j} \operatorname{Re}\left(U_{f i} U_{f^{\prime} i}^{*} U_{f j}^{*} U_{f^{\prime} j} e^{\left[i\left(E_{j}-E_{i}\right) t\right]}\right)
$$

where $E_{i}=\sqrt{p^{2}+m_{i}^{2}}$ is the energy of the mass eigenstate $\tilde{v}_{i}$. Of a particular interest for our case where the active neutrinos oscillates into bulk states is the survival probability after a time $t$, given by

$$
P_{f \rightarrow f}(t)=\left.\left.\left|\sum_{i}\right| U_{f i}\right|^{2} e^{i E_{i} t}\right|^{2} .
$$


We consider in the following the case of the Dirac neutrinos discussed in the previous section. We can explicitly derive the neutrino oscillation probability using the eigenvalues and eigenvalues of the (doubly) infinite neutrino mass matrix in the KK basis. The active neutrino survival probability, in the non-relativistic approximation, is given by the formula $[9,19]$

$$
P_{v_{L} \rightarrow v_{L}}(t)=\left|\sum_{\left(k_{1}, k_{2}\right) \in \mathscr{I}} \frac{2}{N_{\left(k_{1}, k_{2}\right)}} e^{i \frac{m_{\left(k_{1}, k_{2}\right)}^{2}}{2 p}}\right|^{2}=\left|\sum_{k_{1}, k_{2}=-\infty}^{\infty} \frac{2^{\delta_{k_{1} 0} \delta_{k_{2} 0}}}{N_{\left(k_{1}, k_{2}\right)}} e^{i \frac{m_{\left(k_{1}, k_{2}\right)}^{2}}{2 p}}\right|^{2} .
$$

The factor 2 in the first sum accounts for the fact that each massive state corresponds to a Dirac neutrino. An approximate estimation of the normalisation factors $N_{\left(k_{1}, k_{2}\right)}$ gives the result

$$
N_{\left(k_{1}, k_{2}\right)} \simeq \frac{2 \bar{m}^{2}}{m_{\left(k_{1}, k_{2}\right)}^{2}}
$$

where $m_{\left(k_{1}, k_{2}\right)}$ are the KK masses. We can anticipate from (4.5) some similarities and also some differences between the 5D and the present 6D case. Like in the 5D case [9], for large KK masses $N_{i} \sim m_{i}^{2}$ and the active neutrino mostly oscillates into the lowest mass states. In the 6D case, on the other hand, the 'degeneracy' of the massive states is higher than in 5D and the decoupling of the massive states is slower than in 5D. Numerically too, we find similar behaviour. For a relatively small number of states, $P(t)$ is always smaller than 1 . In fact, even $P(0)$ is smaller than 1 , unless one sums over all the states. $P(t)$ oscillates with $t$, though it is always remaining smaller than $P(0)$. The oscillations become more rapid as we sum over more and more states. Finally lets note that the brane Majorana case can simply be recovered from the previous expressions by the replacement $\bar{m}^{2} \rightarrow \bar{m}^{2}+m_{i} M_{0}$.

Further, in order to have sizable running the active neutrinos are required not to be much lighter than the sterile neutrinos, the oscillations into the bulk sterile states are constrained by the existing experimental data. The two flavour active-sterile oscillations can be suppressed by choosing a sufficiently small coupling $h_{2}$. For example, for $h_{2}=0.05$ we find (for $\Lambda=100 \mathrm{GeV}$ ) and summing up to first 200 states that the survival probability of the active neutrinos can stay around $70 \%$. The survival probability obviously decreases as $h_{2}$ increases. When other active flavours are added, since mass differences for active neutrinos $\Delta m_{v_{e} v_{\mu}}^{2}, \Delta m_{v_{e} v_{\tau}}^{2} \ll R_{c}^{-2} \sim e V^{2}$, for small values of the coupling $g_{2}$ and for all KK states the mass differences $m_{v_{i}}^{2}-m_{K K}^{2} \gg \Delta m_{v_{e} v_{\mu}}^{2}, \Delta m_{v_{e} v_{\tau}}^{2}$. In this case, the oscillations into sterile neutrinos are subdominant with respect to active neutrino oscillations.

\section{Discussion}

In this talk, we have presented a model for neutrino mass generation where a 6D Weyl neutrino interacts with the 4D SM neutrinos through a brane localised mass term. We have shown that the brane localised coupling gets renormalised with energy (in the 4D sense) at the tree level. In our work[19], we find this result by two different methods. First by diagonalizing the (infinite) mass matrix in the KK basis, along the lines of $[9,10,22]$. Second by considering the bulk propagation of the fields with appropriate boundary conditions due to the orbifold projection in presence of brane-localized operators [23]. While, most of the discussion has been concentrated for the case of 
a single generation, we can easily extend the analysis presented for more than one flavour. In such a case, we need to introduce three distinct bulk sterile Weyl fermions $\Psi_{a}$ and therefore the branebulk mixing mass term becomes a $3 \times 3$ matrix. It can be then shown that the classical running does not modify the flavour mixing present at the scale $\Lambda$ by renormalising it in evolving towards low energy.

Acknowledgements : I am grateful to my collaborators E. Dudas and C. Grojean without whom this work would not have been done. We are grateful to G. Bhattacharyya, T. Gherghetta, P. Holstein, S. Lavignac, J. Mourad, C. Papineau, M. Peloso, K. Sridhar and especially to V. Rubakov for suggestions and discussions.

\section{References}

[1] N. Arkani-Hamed, S. Dimopoulos and G. R. Dvali, Phys. Lett. B 429, 263 (1998) [arXiv:hep-ph/9803315] ; I. Antoniadis, N. Arkani-Hamed, S. Dimopoulos and G. R. Dvali, Phys. Lett. B 436, 257 (1998) [arXiv:hep-ph/9804398].

[2] I. Antoniadis, Phys. Lett. B 246, 377 (1990).

[3] L. Randall and R. Sundrum, Phys. Rev. Lett. 83, 3370 (1999) [arXiv:hep-ph/9905221] ; Phys. Rev. Lett. 83 (1999) 4690 [arXiv:hep-th/9906064] ; M. Gogberashvili, Int. J. Mod. Phys. D 11 (2002) 1635 [arXiv:hep-ph/9812296].

[4] K. R. Dienes, E. Dudas and T. Gherghetta, Phys. Lett. B 436, 55 (1998) [arXiv:hep-ph/9803466]; K. R. Dienes, E. Dudas and T. Gherghetta, Nucl. Phys. B 537, 47 (1999) [arXiv:hep-ph/9806292].

[5] A. Delgado, A. Pomarol and M. Quiros, JHEP 0001, 030 (2000) [arXiv:hep-ph/9911252]; T. Appelquist, H. C. Cheng and B. A. Dobrescu, Phys. Rev. D 64 (2001) 035002 [arXiv:hep-ph/0012100].

[6] G. F. Giudice, R. Rattazzi and J. D. Wells, Nucl. Phys. B 544, 3 (1999) [arXiv:hep-ph/9811291]; E. A. Mirabelli, M. Perelstein and M. E. Peskin, Phys. Rev. Lett. 82 (1999) 2236 [arXiv:hep-ph/9811337]; T. Han, J. D. Lykken and R. J. Zhang, Phys. Rev. D 59 (1999) 105006 [arXiv:hep-ph/9811350].

[7] Y. Fukuda et al. [Super-Kamiokande Collaboration], Phys. Rev. Lett. 81 (1998) 1562 [arXiv:hep-ex/9807003]; S. Fukuda et al. [Super-Kamiokande Collaboration], Phys. Rev. Lett. 86 (2001) 5651 [arXiv:hep-ex/0103032].

[8] P. Minkowski, Phys. Lett. B 67 (1977) 421 ; M. Gell-Mann, P. Ramond and R. Slansky, in (P. van Nieuwenhuisen and D.Z. Freedman eds.), North-Holland, Amsterdam, 1979; T. Yanagida, in Proceedings of the Workshop on Unified Theories and Baryon Number in the Universe (A. Sawada and A. Sugamoto eds.), KEK preprint 79-18, 1979; R. N. Mohapatra and G. Senjanovic, Phys. Rev. Lett. 44 (1980) 912.

[9] K. R. Dienes, E. Dudas and T. Gherghetta, Nucl. Phys. B 557, 25 (1999) [arXiv:hep-ph/9811428]; K. R. Dienes and I. Sarcevic, Phys. Lett. B 500 (2001) 133 [arXiv:hep-ph/0008144].

[10] N. Arkani-Hamed, S. Dimopoulos, G. R. Dvali and J. March-Russell, Phys. Rev. D 65 (2002) 024032 [arXiv:hep-ph/9811448] ; G. R. Dvali and A. Y. Smirnov, Nucl. Phys. B 563 (1999) 63 [arXiv:hep-ph/9904211].

[11] Y. Grossman and M. Neubert, Phys. Lett. B 474 (2000) 361 [arXiv:hep-ph/9912408]; T. Gherghetta and A. Pomarol, Nucl. Phys. B 586 (2000) 141 [arXiv:hep-ph/0003129]. 
[12] R. N. Mohapatra, S. Nandi and A. Perez-Lorenzana, Phys. Lett. B 466 (1999) 115 [arXiv:hep-ph/9907520]; A. Ioannisian and A. Pilaftsis, Phys. Rev. D 62 (2000) 066001 [arXiv:hep-ph/9907522]; R. Barbieri, P. Creminelli and A. Strumia, Nucl. Phys. B 585 (2000) 28 [arXiv:hep-ph/0002199]; A. Lukas, P. Ramond, A. Romanino and G. G. Ross, Phys. Lett. B 495 (2000) 136 [arXiv:hep-ph/0008049] and JHEP 0104 (2001) 010 [arXiv:hep-ph/0011295]; A. De Gouvea, G. F. Giudice, A. Strumia and K. Tobe, Nucl. Phys. B 623, 395 (2002) [arXiv:hep-ph/0107156]; G. Bhattacharyya, H. V. Klapdor-Kleingrothaus, H. Paes and A. Pilaftsis, Phys. Rev. D 67 (2003) 113001 [arXiv:hep-ph/0212169].

[13] I. Antoniadis, K. Benakli and M. Quiros, New J. Phys. 3, 20 (2001) [arXiv:hep-th/0108005]; C. Csáki, C. Grojean and H. Murayama, Phys. Rev. D 67, 085012 (2003) [arXiv:hep-ph/0210133] ; G. von Gersdorff and M. Quiros, Phys. Rev. D 68 (2003) 105002 [arXiv:hep-th/0305024].

[14] T. Appelquist, B. A. Dobrescu, E. Ponton and H. U. Yee, Phys. Rev. Lett. 87, 181802 (2001) [arXiv:hep-ph/0107056].

[15] B. A. Dobrescu and E. Poppitz, Phys. Rev. Lett. 87, 031801 (2001) [arXiv:hep-ph/0102010].

[16] G. Servant and T. M. P. Tait, Nucl. Phys. B 650 (2003) 391 [arXiv:hep-ph/0206071].

[17] T. Asaka, W. Buchmuller and L. Covi, Phys. Lett. B 523, 199 (2001) [arXiv:hep-ph/0108021] and Nucl. Phys. B 648, 231 (2003) [arXiv:hep-ph/0209144]; L. J. Hall, Y. Nomura, T. Okui and D. R. Smith, Phys. Rev. D 65 (2002) 035008 [arXiv:hep-ph/0108071].

[18] T. Appelquist, B. A. Dobrescu, E. Ponton and H. U. Yee, Phys. Rev. D 65, 105019 (2002) [arXiv:hep-ph/0201131]; J. Matias and C. P. Burgess, arXiv:hep-ph/0508156.

[19] E. Dudas, C. Grojean and S. K. Vempati, arXiv:hep-ph/0511001.

[20] H. Georgi, A. K. Grant and G. Hailu, Phys. Lett. B 506 (2001) 207 [arXiv:hep-ph/0012379].

[21] W. D. Goldberger and M. B. Wise, Phys. Rev. D 65 (2002) 025011 [arXiv:hep-th/0104170].

[22] B. A. Dobrescu and E. Ponton, JHEP 0403, 071 (2004) [arXiv:hep-th/0401032]; G. Burdman, B. A. Dobrescu and E. Ponton, arXiv:hep-ph/0506334.

[23] C. Csáki, C. Grojean, H. Murayama, L. Pilo and J. Terning, Phys. Rev. D 69, 055006 (2004) [arXiv:hep-ph/0305237]; C. Csáki, C. Grojean, L. Pilo and J. Terning, Phys. Rev. Lett. 92, 101802 (2004) [arXiv:hep-ph/0308038];Z. Chacko, M. Graesser, C. Grojean and L. Pilo, Phys. Rev. D 70, 084028 (2004) [arXiv:hep-th/0312117]; G. Cacciapaglia, C. Csáki, C. Grojean, M. Reece and J. Terning, arXiv:hep-ph/0505001. 